\title{
Camrelizumab monotherapy leading to partial remission for relapsed upper tract urothelial carcinoma after radical nephroureterectomy: a case report
}

\author{
Kangxin Ni, Zhenghui Wang, Shicheng Yu, Jintong Zheng, Gonghui Li \\ Department of Urology, Sir Run Run Shaw Hospital, Zhejiang University School of Medicine, Hangzhou, China \\ Correspondence to: Gonghui Li. Department of Urology, Sir Run Run Shaw Hospital, Zhejiang University School of Medicine, No.3 East Qingchun \\ Road, Hangzhou 310016, China. Email: 3193119@zju.edu.cn.
}

\begin{abstract}
Upper tract urothelial carcinoma (UTUC) is a rare malignant disease, and while locally advanced non-metastatic UTUC can be cured by radical nephroureterectomy (RNU), this procedure leaves patients at high risk of relapse and death from cancer. Though the FDA has currently approved five agents for the systemic immunotherapy treatment of urothelial carcinoma (UC) patients, the effect of immunotherapy in patients with recurrent UTUC still lacks specific evidence. Camrelizumab is a programmed cell death protein 1 (PD-1) inhibitor which has been approved for the treatment of recurrent or refractory classical Hodgkin lymphoma in China and have achieved improvement in a verity of solid tumors with manageable safety profile. We herein report a case of an 80-year-old woman diagnosed with localized UTUC (pT4N0M0) for which she underwent RNU but relapsed after 2 months. As the toxic effects of chemotherapy were intolerable for the patient, she received the PD-1 inhibitor Camrelizumab as a salvage treatment to stop tumor growth. The tumor shrank and the patient achieved partial response (PR) after eight cycles but progressed after 14 cycles. Based on the current evidence, our case indicated that Camrelizumab is a promising agent in treating locally advanced and recurrent UTUC patients with poor performance status and imparted renal function.
\end{abstract}

Keywords: Upper tract urothelial carcinoma (UTUC); Camrelizumab (SHR-1210); immune checkpoint inhibitor (ICI); programmed cell death protein 1 (PD-1); case report

Submitted Feb 26, 2021. Accepted for publication Apr 20, 2021.

doi: $10.21037 /$ tau-21-268

View this article at: http://dx.doi.org/10.21037/tau-21-268

\section{Introduction}

Upper tract urothelial carcinoma (UTUC) is a relatively rare malignant disease accounting for $5-10 \%$ of urothelial carcinoma (UC) and occurring in roughly two people per 100,000 (1). The epidemiological risk factors associated with UTUC include smoking, carcinogenic aromatic amines, aristolochic acid and Lynch syndrome (2). As UTUC is highly aggressive, nearly $60 \%$ of cases are invasive at time of diagnosis (3). While radical nephroureterectomy (RNU) remains the gold standard treatment for nonmetastatic high risk UTUC (4), approximately $25 \%$ of patients experience a recurrence or metastasis after RNU, which is associated with $\mathrm{T}$ classification, lymph node metastasis, lymphovascular invasion, sessile tumor architecture and concomitant carcinoma in situ. While gender is no longer considered a prognostic factor in UTUC patients, ureteroscopy before RNU lead to a significantly increased risk of intravesical recurrence in patients with UTUC $(2,5)$. Although salvage chemotherapy (SC) can provide a survival benefit for patients with recurrent UTUC (6), most patients are ineligible to receive it due to impaired performance status and renal dysfunction.

Checkpoint inhibitors are a new treatment option for UTUC patients, and their requirement for renal function is relatively low. The molecular pathways of potential drug targets for UTUC include CTLA4, PD1 and PDL1 (2). 
To date, five immune checkpoint inhibitors (ICIs) (atezolizumab, pembrolizumab, nivolumab, durvalumab, and avelumab) have already approved by the Food and Drug Administration (FDA) of the USA based on the results of clinical trials (7). However, specific evidence of immunotherapy for UTUC is still lacking as most patients enrolled in these trials had UCs of the bladder inside the upper tract. Camrelizumab is a programmed cell death protein 1 (PD-1) inhibitor which has received approval in China for the treatment of recurrent or refractory classical Hodgkin lymphoma (8). However, its efficacy in UTUC has not been reported. Herein, we present a case of relapsed UTUC with high PD-1 expression treated by single Camrelizumab, resulting in a partial response (PR) confirmed by CT images. We present the following case in accordance with the CARE Reporting checklist (available at http://dx.doi.org/10.21037/tau-21-268).

\section{Case presentation}

An 80-year-old Chinese woman, with a medical history of hypertension and diabetes for 20 years was admitted to our hospital in October 2019 due to right waist soreness in the preceding 5 months and lower abdominal distension in the preceding 4 months. Physical examination showed that the superficial lymph nodes were not enlarged, there was no tenderness or rebound tenderness in the entire abdomen, and no obvious percussion tenderness in the renal area. However, contrast-enhanced abdominal computed tomography (CT) showed right renal atrophy with hydronephrosis and thickening of the pelvic part of the right ureter (Figure 1A), and the patient was diagnosed with right ureter cancer $\left(\mathrm{T}_{2} \mathrm{~N}_{0} \mathrm{M}_{0}\right)$.

On October 24, 2019, the patient received robot-assisted RNU. Postoperative pathology revealed a high-grade invasive UC, and the outer membranous layer of the ureter had been invaded. As the patient was of advanced age and had impaired renal function, neoadjuvant chemotherapy (NAC) treatment was not conducted after RNU. On October 27, 2019, laboratory findings reported a serum creatinine concentration of $117 \mu \mathrm{mol} / \mathrm{L}$ and contrastenhanced abdominal CT showed a recurrent tumor mass in the inferior segment of the right ureter which had invaded surrounding tissue with a diameter of $36 \mathrm{~mm}\left(\mathrm{pT}_{4} \mathrm{~N}_{0} \mathrm{M}_{0}\right)$ (Figure 1B).

Given the tumor was unresectable and the patient was ineligible for chemotherapy due to their advanced age and renal dysfunction, genetic testing was conducted. As the results showed strong-positive PD-L1 expression and high tumor mutation burden (TMB), salvage Camrelizumab (200 mg once, every 3 weeks) was initiated. After five cycles of immunotherapy, the longest diameter of the tumor had decreased from 36 to $24 \mathrm{~mm}$ (Figure 1C) and after eight cycles, abdomen CT revealed this had decreased from 24 to $15 \mathrm{~mm}$, indicating a PR according to the Response Evaluation Criteria in Solid Tumors (RECIST) classification (Figure 1D).

Despite this, after 11 cycles of immunotherapy, abdomen CT revealed a greater tumor diameter in the inferior segment of the right ureter (Figure 1E), and after another three treatment cycles, the longest diameter of the tumor mass had increased to $30 \mathrm{~mm}$, indicating disease progression (Figure $1 F$ ). Throughout the treatment course, the patient experienced skin rash which was relieved after symptomatic treatment. At the time of writing, the patient is receiving combined treatment consisting of Camrelizumab and Anlotinib, and follow-up is ongoing (Figure 2).

Written informed consent was obtained from the patient before publication of this case report. All procedures performed in studies involving human participants were in accordance with the ethical standards of the institutional and/or national research committee(s) and with the Helsinki Declaration (as revised in 2013) (9).

\section{Discussion}

While RNU is the gold standard treatment for patients with nonmetastatic high-risk UTUC (10), the therapeutic benefits of lymph node dissection at the time of RNU remains controversial (2), and the rate of recurrence is high (4). Previous studies indicated similar survival after kidney-sparing surgery (KSS) versus RNU in patients with low-grade and noninvasive UTUC, but high-quality evidence of the different clinical outcomes between KSS and RNU in patients with high-grade UTUC is still lacking (11). For locally advanced UTUC patients, NAC treatment may provide better survival outcomes compared with single RNU (12), and SC was associated with improved survival in patients with recurrent UTUC (6). However, elderly patients tend to become ineligible for chemotherapy due to a decline in renal function after RNU.

In comparison, the requirement for renal function is lower in immunotherapy than in chemotherapy. Between 2016 to 2020, five ICIs including atezolizumab, nivolumab, pembrolizumab, avelumab, and durvalumab were approved by the FDA in treating advanced or metastatic 
A

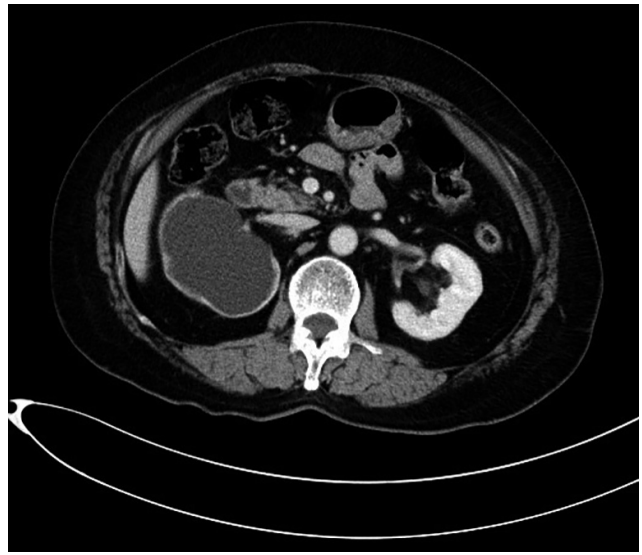

B

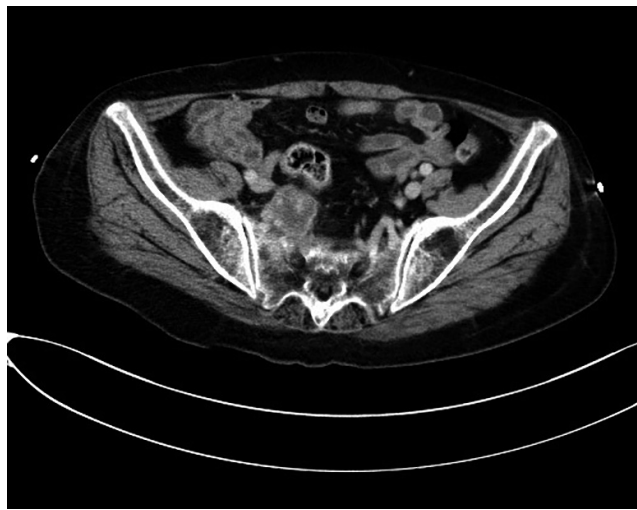

C

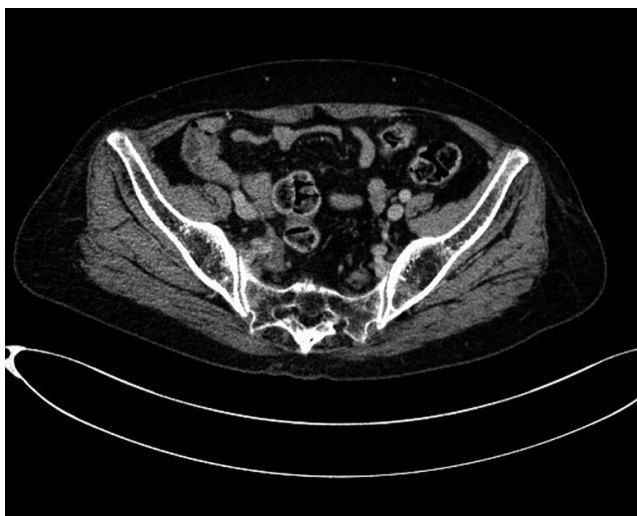

D

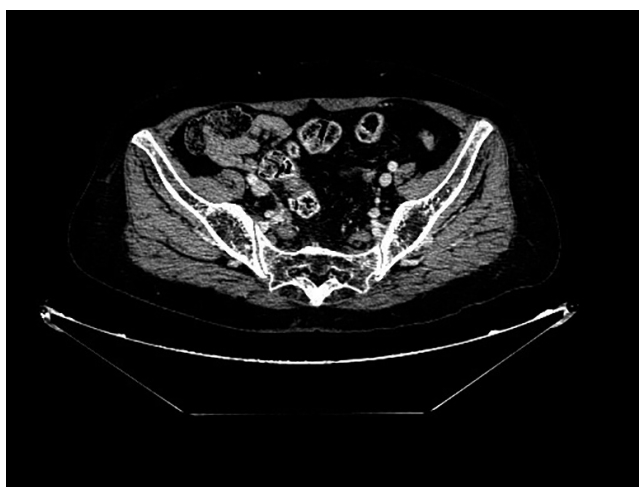

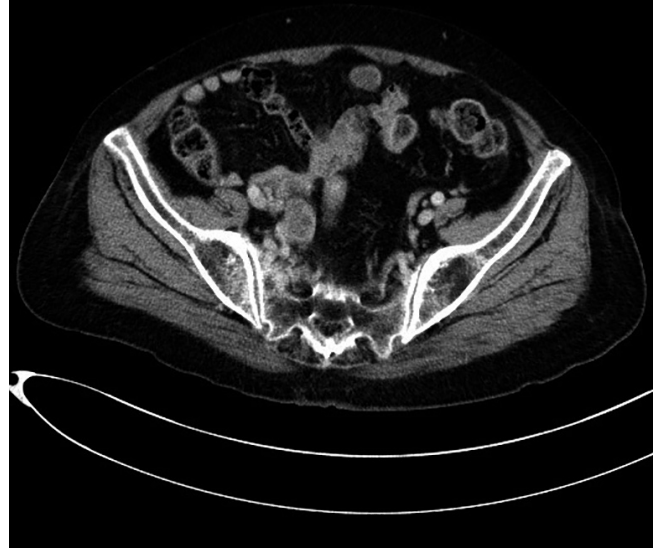
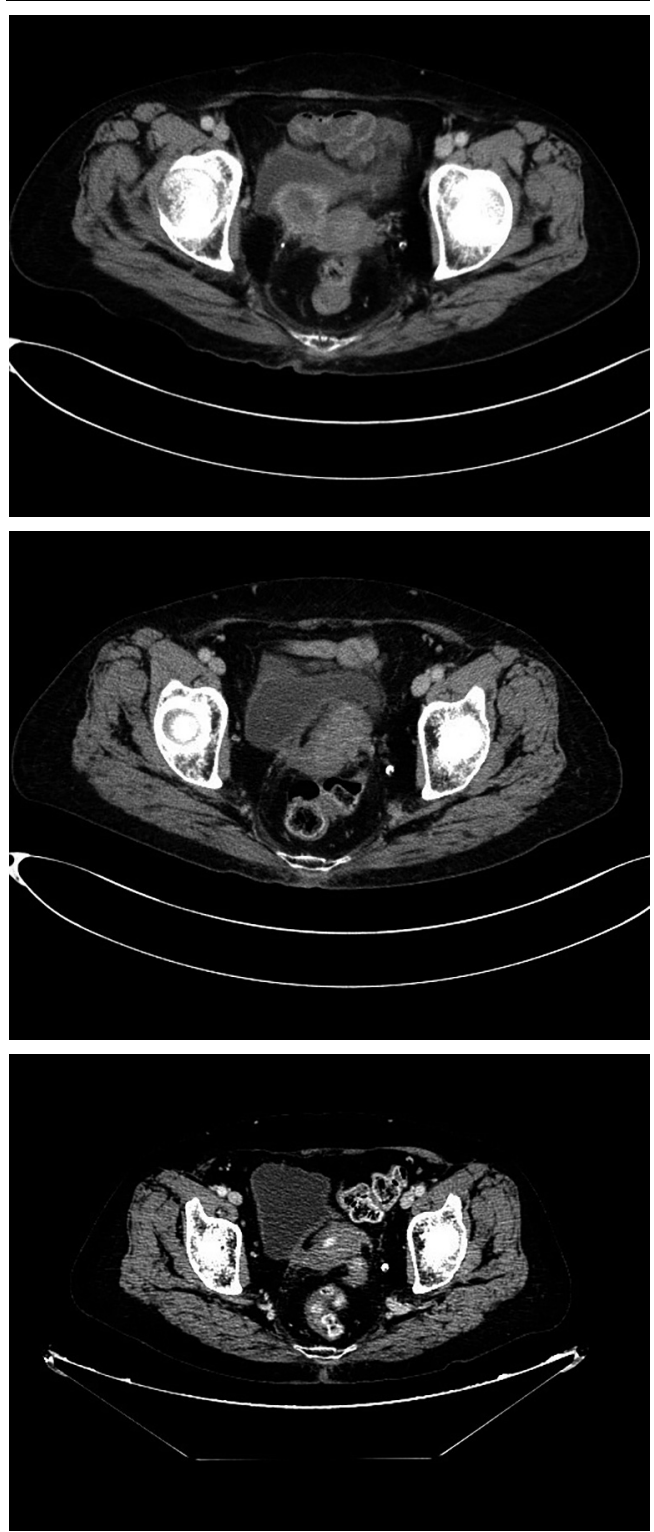

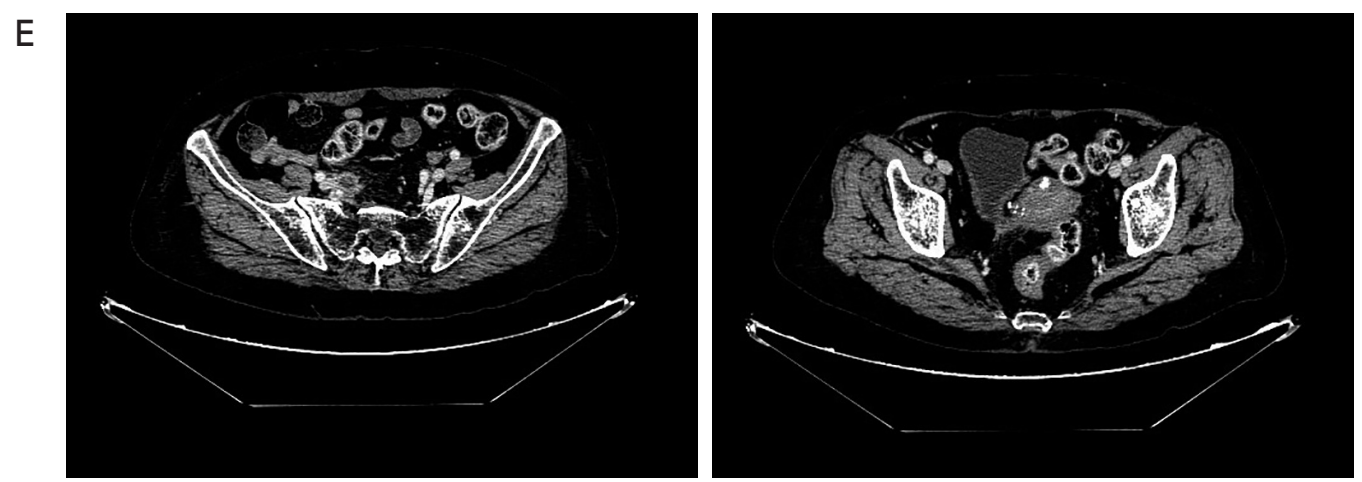

F
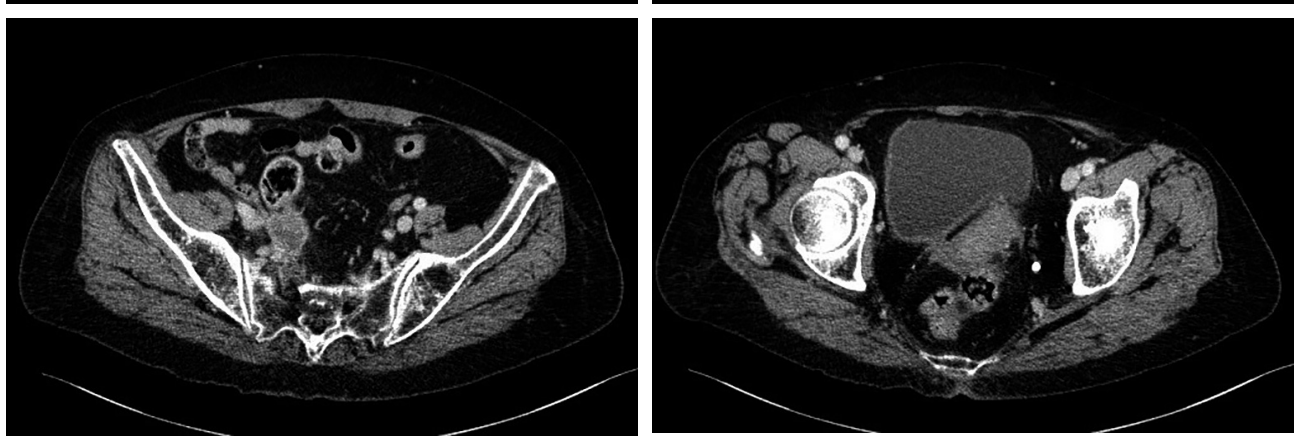

Figure 1 Abdominal CT images throughout the period: (A) UTUC before RNU, (B) recurrent UTUC after RNU, and recurrent UTUC after receiving single Camrelizumab treatment for (C) 5 cycles, (D) 8 cycles, (E) 11 cycles, (F) 14 cycles. UTUC, upper tract urothelial carcinoma; RNU, radical nephroureterectomy.

$2019 / 10$

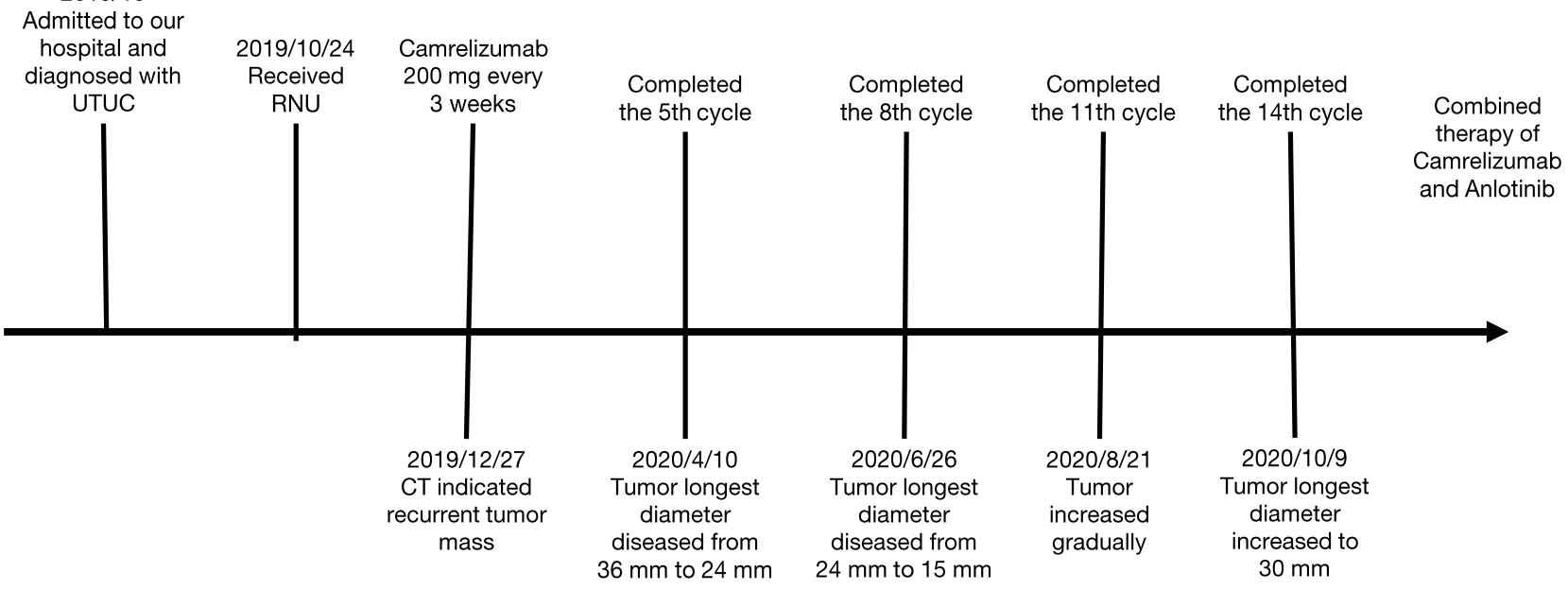

Figure 2 Timeline picture of this case. UTUC, upper tract urothelial carcinoma; RNU, radical nephroureterectomy.

UC patients (7). However, the data on immunotherapy for UTUC patients are limited and are often deduced form studies enrolling UC patients.

A meta-analysis showed patients with refractory UC can benefit from PD-1 and PD-ligand 1 (PD-L1) inhibitors, and the response rate is associated with $\mathrm{PD}-\mathrm{L} 1$ expression and TMB (13). Additionally, several studies with PD-1 or PD-L1 inhibitors as first-line treatment for locally 
advanced and unresectable or metastatic UC demonstrated encouraging results in response rate and toxic effect $(14,15)$.

The incidence rate of PD-L1 expression in UTUC patients was approximately $13.0-30.0 \%$ and PD-L1 overexpression related to a more aggressive nature (16) and shorter OS in UTUC patients after RNU (17). However, the relationship between PD-L1 expression and response to therapy with PD-L1 inhibitors remains unclear.

In the present case, because of impaired renal function and poor performance status, the patient received single Camrelizumab treatment for relapsed UTUC inside of chemotherapy. During the treatment, the patient only experienced manageable toxicity and achieved a PR after eight cycles, which is consistent with those of previous investigations. Though there are currently no clinical trials focus on the effect of PD-1 in patients with UTUC, several single case studies have been reported in which durable response have been gained in patients with UTUC $(18,19)$. The present study has several limitations, including a short follow-up time and being a single case.

In summary, this case report showed that single Camrelizumab may provide an alternative option for patients with recurrent UTUC after RNU, and there may be a positive connection between PD-L1 expression and the response rate to therapy with $\mathrm{PD}-\mathrm{L} 1$ inhibitors.

\section{Conclusions}

To the best of our knowledge, this is the first report of a PD-L1 positive UTUC patient treated by single Camrelizumab after relapse who achieved a PR, which may provide an additional option for patients with recurrent UTUC.

\section{Acknowledgments}

Funding: None.

\section{Footnote}

Reporting Checklist: The authors have completed the CARE reporting checklist. Available at http://dx.doi.org/10.21037/ tau-21-268

Conflicts of Interest: All authors have completed the ICMJE uniform disclosure form (available at http://dx.doi. org/10.21037/tau-21-268). The authors have no conflicts of interest to declare.
Ethical Statement: The authors are accountable for all aspects of the work in ensuring that questions related to the accuracy or integrity of any part of the work are appropriately investigated and resolved. Written informed consent was obtained from the patient for publication of this study and any accompanying images. All procedures performed in studies involving human participants were in accordance with the ethical standards of the institutional and/or national research committee(s) and with the Helsinki Declaration (as revised in 2013).

Open Access Statement: This is an Open Access article distributed in accordance with the Creative Commons Attribution-NonCommercial-NoDerivs 4.0 International License (CC BY-NC-ND 4.0), which permits the noncommercial replication and distribution of the article with the strict proviso that no changes or edits are made and the original work is properly cited (including links to both the formal publication through the relevant DOI and the license). See: https://creativecommons.org/licenses/by-nc-nd/4.0/.

\section{References}

1. Rouprêt M, Babjuk M, Burger M, et al. European Association of Urology Guidelines on Upper Urinary Tract Urothelial Carcinoma: 2020 Update. Eur Urol 2021;79:62-79.

2. Soria F, Shariat SF, Lerner SP, et al. Epidemiology, diagnosis, preoperative evaluation and prognostic assessment of upper-tract urothelial carcinoma (UTUC). World J Urol 2017;35:379-87.

3. Qiu D, Hu J, He T, et al. Effect of neoadjuvant chemotherapy on locally advanced upper tract urothelial carcinoma: a pooled analysis. Transl Androl Urol 2020;9:2094-106.

4. Margulis V, Shariat SF, Matin SF, et al. Outcomes of radical nephroureterectomy: a series from the Upper Tract Urothelial Carcinoma Collaboration. Cancer 2009;115:1224-33.

5. Kenigsberg AP, Meng X, Ghandour R, et al. Oncologic outcomes of radical nephroureterectomy (RNU). Transl Androl Urol 2020;9:1841-52.

6. Ikeda M, Matsumoto K, Hirayama T, et al. Oncologic Outcomes of Salvage Chemotherapy in Patients with Recurrent or Metastatic Lesions after Radical Nephroureterectomy: A Multi-Institutional Retrospective Study. Chemotherapy 2020;65:134-40.

7. Baldini C, Champiat S, Vuagnat P, et al. Durvalumab for 
the management of urothelial carcinoma: a short review on the emerging data and therapeutic potential. Onco Targets Ther 2019;12:2505-12.

8. Markham A, Keam SJ. Camrelizumab: First Global Approval. Drugs 2019;79:1355-61.

9. Gagnier JJ, Kienle G, Altman DG, et al. The CARE Guidelines: Consensus-based Clinical Case Reporting Guideline Development. Glob Adv Health Med 2013;2:38-43.

10. Leow JJ, Liu Z, Tan TW, et al. Optimal Management of Upper Tract Urothelial Carcinoma: Current Perspectives. Onco Targets Ther 2020;13:1-15.

11. Seisen T, Peyronnet B, Dominguez-Escrig JL, et al. Oncologic Outcomes of Kidney-sparing Surgery Versus Radical Nephroureterectomy for Upper Tract Urothelial Carcinoma: A Systematic Review by the EAU Non-muscle Invasive Bladder Cancer Guidelines Panel. Eur Urol 2016;70:1052-68.

12. Necchi A, Lo Vullo S, Mariani L, et al. Adjuvant chemotherapy after radical nephroureterectomy does not improve survival in patients with upper tract urothelial carcinoma: a joint study by the European Association of Urology-Young Academic Urologists and the Upper Tract Urothelial Carcinoma Collaboration. BJU Int 2018;121:252-9.

13. Tafuri A, Smith DD, Cacciamani GE, et al. Programmed Death 1 and Programmed Death Ligand 1 Inhibitors in Advanced and Recurrent Urothelial Carcinoma: Metaanalysis of Single-Agent Studies. Clin Genitourin Cancer 2020;18:351-60.e3.

14. Balar AV, Galsky MD, Rosenberg JE, et al. Atezolizumab as first-line treatment in cisplatin-ineligible patients with locally advanced and metastatic urothelial carcinoma: a single-arm, multicentre, phase 2 trial. Lancet 2017;389:67-76.

15. Balar AV, Castellano D, O'Donnell PH, et al. First-line pembrolizumab in cisplatin-ineligible patients with locally advanced and unresectable or metastatic urothelial cancer (KEYNOTE-052): a multicentre, single-arm, phase 2 study. Lancet Oncol 2017;18:1483-92.

16. Skala SL, Liu TY, Udager AM, et al. Programmed Deathligand 1 Expression in Upper Tract Urothelial Carcinoma. Eur Urol Focus 2017;3:502-9.

17. Lu Y, Kang J, Luo Z, et al. The Prevalence and Prognostic Role of PD-L1 in Upper Tract Urothelial Carcinoma Patients Underwent Radical Nephroureterectomy: A Systematic Review and Meta-Analysis. Front Oncol 2020;10:1400.

18. Ding X, Zong J, Li X, et al. Dramatic responses of recurrent upper urinary tract urothelial carcinoma harboring FGFR3 and TP53 activating mutations to Pembrolizumab in combination with Erdafitinib: A Case Report. Onco Targets Ther 2021;14:2177-83.

19. Ikarashi D, Kitano S, Ishida K, et al. Complete pathological response to neoadjuvant Pembrolizumab in a patient with chemoresistant upper urinary tract urothelial carcinoma: a case report. Front Oncol 2020;10:564714.
Cite this article as: Ni K, Wang Z, Yu S, Zheng J, Li G. Camrelizumab monotherapy leading to partial remission for relapsed upper tract urothelial carcinoma after radical nephroureterectomy: a case report. Transl Androl Urol 2021;10(4):1821-1826. doi: 10.21037/tau-21-268 